\title{
The Internet of Healthy Things
}

\author{
Jung A Kim, RN, PhD
}

College of Nursing, Hanyang University, Seoul, Korea

joyhippo@hanyang.ac.kr

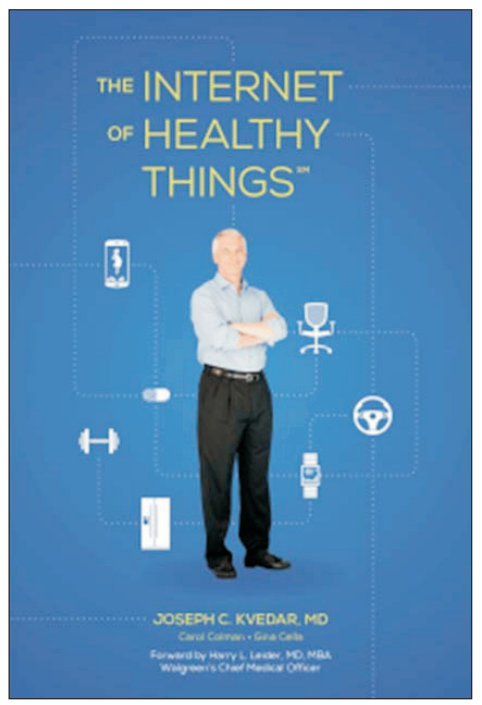

Author: Joseph C. Kvedar

Year: 2015

Name and location of publisher: Partners Connected

Health, Boston (MA)

Number of pages: 270

Language: English

ISBN: 978-0692534571

This is an Open Access article distributed under the terms of the Creative Commons Attribution Non-Commercial License (http://creativecommons.org/licenses/bync/4.0/) which permits unrestricted non-commercial use, distribution, and reproduction in any medium, provided the original work is properly cited.

(C) 2016 The Korean Society of Medical Informatics
The world now faces the new era of the Internet of Things (IoT). The acronym IoT was coined in 1999 by Ashton [1], referring to the "networked interconnection of everyday objects, which are often equipped with ubiquitous intelligence" [2]. Currently, the number of devices connected to each other exceeds the entire population of the world, and such change has converted the concept of the internet from an "internet between people" to an "internet between things" [3].

The expansion of the IoT has resulted in changes in the healthcare service area as well. Through IoT technologies, the biometric data of consumers are automatically captured in real time and sent to primary care professionals, who provide recommendations and coaching for health and wellness based on the unique data streams delivered from powerful offsite computers. This process will ultimately make consumers' lifestyles, daily activities, and health behavior more healthy $[4,5]$. Such changes are currently in progress, and Kvedar [4] now refer to the Internet of Healthy Things to explain such phenomenon.

Even prior to the existence of the internet, smartphones, apps and sensors, Dr. Kvedar claimed that healthcare should move from the hospital and the physician's office to the everyday lives of patients. He implemented personal health technologies with which the patient and healthcare provider could improve the management of chronic diseases, maintain health and wellness, and improve clinical outcomes in the healthcare delivery system [6]. Based on his experience as a doctor and a pioneer in the area of digital healthcare for more than 20 years, he summarized the knowledge, experience, and lessons that he gained in a book entitled, "The Internet of Healthy Things." This book does not simply describe the ideal future of healthcare but instead discusses actual attempts that have been and are being carried out, while also discussing frankly the obstacles and challenges 
that faced during such a process. Readers of this book can share Kvedar's deep insight in the areas of IoT and digital health, actual cases he experienced during his long career, and the wisdom that he gained through such experiences. This book is very helpful in that it clearly presents to readers the concepts of the connection between healthcare and digital technology as well as the relevant healthcare policies and regulations, which can be perplexing to the average reader.

This book consists of a foreword, 12 chapters, and an afterword. The chapters are summarized below.

Chapter 1 (20/20 Foresight): In the first chapter of his book, he discusses an average day, specifically October 30, 2020. During the day, which starts when a person wakes up from the voice of their virtual health coach, seamless health management and health promotion activities are carried out in all places and at all times.

Chapter 2 (Seeing Around Corners): The author explains what he imagines with regard to future health and health system over the past 20 years, the history through which imagination became reality, and why health systems should focus on IoT. The author notes that our current situation is only the beginning and introduces predictions by experts who contend that it will be possible to collect, receive and share data by connecting to a global network through inexpensive sensors, GPS and cloud computing with 26 billion everyday objects by 2020 . Such biometric data are automatically captured in real time, and the impact of the data on human lifestyles, diseases, and wellness and behavior will be identified, with the results then used to transform human behavior. He also explains that his team coined the term Internet of Healthy Things (IoHT) to refer to such phenomena.

Chapter 3 (The Big Shakeup): This chapter explains the status of, and problems associated with, the current payment system, and it narrates the need for a new payment system for the age of the IoHT, along with relevant models. It also discusses the possibility of implementing a new system in reality as well as potential barriers to this.

Chapter 4 (The Hardest Sell): He claims that providing new technologies to people does not guarantee that they will use such technologies. He explains why people who need the IoHT may not focus on the IoHT, why they may not use it, and strategies to overcome such gaps.

Chapter 5 (The New White Coat Anxiety): The author states that in the age of the IoHT, the roles of doctors and nurses will change and that they will have fear and resist converting to the new roles for which they have no education or training, and have never experienced. However, he emphasizes that the IoHT will present even more opportuni- ties to doctors and nurses, reminding us also that change is inevitable.

Chapter 6 (Some Healthy Disruption): The healthcare system in the United States implements the IoHT and is changing using various methods used to provide "consumer-centric experiences." The author uses an example of such changes is the Veterans Health Administration (VHA), the largest healthcare provider in the United States, and Walgreens, the largest pharmacy chain in the country.

Chapter 7 (Up Close and Hyperpersonal): At present, doctors question patients and patients respond so as to identify their needs. However, patient needs that the doctor may feel are identified are largely based on the doctor's past experiences or on the literature, while compared to self-reported data by patients, objective data used by doctors to manage patients are much more valuable for improving patient health. In the future, rather than asking questions to elicit expected answers from patients, it will be possible to collect and analyze objective data from patients in real time through the IoHT to determine their needs and then to deliver interventions with methods tailored to individual patients immediately.

Chapter 8 (Try a Little Dopamine): Why do people become so easily addicted to their smartphones but not easily addicted to the health apps that they download to their smartphones? The author researched this issue at length and devised three strategies and three tactics.

Chapter 9 (Making Data Actionable): Big data, predictive analytics, and machine learning are the hottest keywords in our modern society. There are high expectations regarding the capabilities and potential associated with these concepts in the healthcare service area as well, and there is considerable progress to posit ways to use them as well. However, he claims that we have not yet been able sufficiently to maximize their value and that we should think about how to integrate the outcomes from these concepts into healthcare and use them to understand human behavior. This chapter also introduces actual issues and their limitations.

Chapter 10 (The Reinvention of Big Pharma): This chapter explains how software tools can have equal or greater effects than some medications in the current era of mobile and ubiquitous connectivity. The author claims that through the IoHT, the patients' conditions are already being precisely sensed. Moreover, the IoHT can monitor the medicines, dosages, instructions, and effects of the medicines that patients should take, thus realizing a great advance. He provides various examples as well.

Chapter 11 (The Digital Rx): There is almost no possibil- 
ity that chronic diseases such as diabetes, obesity and heart disease will be cured simply by taking a pill in our lifetime. More effective methods such as improved diets, sufficient sleep, increasing physical activity, and maintaining an appropriate weight are often too difficult to maintain in reality. In other words, he claims that in order to conquer lifestylerelated diseases, compared to molecular therapeutics, digital therapeutics or digital $\mathrm{Rx}$ will be more effective at promoting recovery and in helping people maintain a healthy lifestyle with the help of the IoHT.

Chapter 12 (The Privacy Trade-Offs): In the digital era, healthcare consumer data can easily be stolen and misused. Personal rights with regard to one's health data must be protected, and consumers should be able to control their data and know exactly how this information is being used. Consumer privacy must be protected thoroughly. Privacy is not a complex issue; it is a simple issue, and he claims that it is an issue that requires honesty by app developers and operators, healthcare providers, and healthcare organizations.

In the last part of the book entitled "What's Next" as the afterword, he reemphasizes the six trends related to IoHT.

Through this book, he claims that the IoHT is a very smart and effective method for delivering healthcare to consumers and that it has enormous potential to tell consumers when and how healthcare can be delivered most effectively. Furthermore, while there are issues that require improvement, such as the obstacles which must be overcome when implementing and utilizing the IoHT, he is confident that the number of healthcare providers, healthcare professionals and consumers, as well as their fields, will continue to grow.

This book is expected to provide very vivid and realistic information to students and scientists who are interested in the onset of the IoHT, records of its development, current applications, and its outcomes, as well as to healthcare providers who see the advent of the age of the IoHT as a new possibility. Furthermore, anyone who is interested in the world of the IoHT, where customers who traditionally played a passive role in healthcare services can enjoy a more active role in maintaining and promoting their health and wellness with the help of the IoHT, will definitely find this book entertaining. This is true because there is nobody in the world who is not interested in their health and wellness or who wants someone else, including healthcare experts, to have complete control over their health and wellness.

\section{References}

1. Ashton K. That 'internet of things' thing. RFiD J 2009;22(7):97-114.

2. Xia F, Yang LT, Wang L, Vinel A. Internet of things. Int J Commun Syst 2012;25(9):1101-2.

3. Choi JH, Antoine BS, Kim JH. Trends of the converging technologies for healthcare and internet of things. J Korean Inst Commun Sci 2014;31(12):10-6.

4. Kvedar JC. The internet of healthy things. 1st ed. Boston (MA): Partners Connected Health; 2015.

5. Vitalari NP. Prospects for the future of the us healthcare industry: a speculative analysis. Am J Med Res 2016;3(2):7-52.

6. Partners Connected Health. The Internet of Healty Things [Internet]. Boston (MA): Partners Connected Health; c2016 [cited at 2016 Jun 23]. Available from: http://theinternetofhealthythings.com/. 